dilated, but there was no strabismus-a circumstance worthy of great attention. The râles heard in both lungs could be explained away by admitting the existence of pulmonary conges. tion associated with tubercle. Notwithstanding these circum. stances, and by reason of the age of the child, the general appearance and the peculiar symptoms already referred to, the diagnosis was more clearly in favour of acute hydrocephalus. The results of the autopsy showed, however, that there was no organic lesion of the brain, and that the case was unmistakably one of typhoid fever.

LARGE RECURRENT TUMOUR OF THE LOWER JAW; REMOVAL; RAPID RECOVERY.

(Under the care of Dr. Grealdis.)

$\mathrm{R}-$, aged fourteen, was admitted into the wards in June last. She is a strong, well-built girl, and enjoys habitual good health.

History and description of the tumour.-About the end of February last the patient became aware of the presence of a tumour situated in the flor of the mouth beneath the tip of the tongue and behind the dental arcade. The tumour was then of the size of a nut. By the month of April following the morbid growth had much increased in size, and had become very prominent in the mouth; a large portion of it was therefore excised by a medical man. At the time of the patient's admission into the hospital the tumour was the size of a small apple, and bulged under the tongue. In that situation it presented an ulcerated but not a bleeding surface, which did not communicate any offensive odour to the breath. The bulging of the tumour could be felt with the finger in the subhyoid region, but did not cause any marked visible deformity. The tumour was immovable, and seemed to adhere to the posterior surface of the body of the lower jaw-bone. On exploring the anterior surface of the bone, it was found to be in a normal condition. The anterior surface of the dental arcade also presented a normal appearance. Immediately behind the teeth could be felt the bulging of the tumour. The lower border of the body of the inferior maxillary was somewhat thickened on the left up to the situation of the ascending ramus; on the right the thickening extended somewhat further. The growth was of hard and elastic consistence.

By July 30 th, at which date the operation was performed, the tumour had rapidly increased to double its size since the time of the patient's admission; but it was particularly the vertical diameter which had augmented. The bulging in the mouth was much more considerable, and the growth now produced a notable deformity of the sub-hyoid region. In front of the central portion of the lower jaw-bone slight bulging had become manifest. The general condition of the patient was still excellent. There was no tumefaction of the ganglia.

Operation. - M. Giraldès resected the body of the submaxillary bone with a portion of its left ascending branch, and removed the tumour, which was well circumscribed and implanted in the bone. A vertical incision was first made, commencing at three centimetres beneath the lower lip, and extending to the middle of the sub-hyoid region. Two lateral incisions were then made, the left extending to the ascending ramus, the right extending to two centimetres within the ascending ramus. Ligature of the facial artery, which was extremely small, was performed. The flaps having been carefully dissected, the resection of the bone was made at about the middle of the ascending ramus on the left side, and at the junction of the body of the bone with the ascending ramus on the right side. The flaps were united by means of a metallic suture. There was no difficulty in separating the tumour from the mylo-hyoid muscles.

The local sequelæ of the wound were of a most favourable kind. The after-treatment consisted mainly in injecting a solution of carbolic acid into the mouth, so as to remove the large quantity of sanious and purulent matter which accumulated there, and in applying to the wound compresses steeped in camphorated alcohol, or carbolic acid, proper restorative diet being administered to the patient. 'The recovery was remarkably rapid, and eight days only after the operation the patient was walking about the wards in high spirits and excellent condition.

Examination of the tumour after removal. - The morbid growth had obviously taken rise in the periosteum ; osseous, needle-like processes were observed proceeding from the maxillary bone, and penetrating into the tissue of the tumnur. The body of the bone was slightly hypertrophied. When cut through, the tumour presented a greyish-white appearance. Its consistence was rather firm, and the tissue did not creak under the scalpel. Examination with the microscope showed that the growth belonged to the now well-defined group of sarcomata. The presence of some cartilaginous and of some osseous elements gave a mixed character to the tumour, of the composition of which some idea may be had by the appellation "osteo-chondro-sarcoma." The sarcoma in the present case belonged to the variety of sarcomata having large fusiform cellules, and did not warrant so gloomy a prognosis as if it had been a medullary sarcoma.

Inoculations with the tumour were performed by M. Joffroy (one of the internes of the service) upon two white mice. Two inoculations were made under the skin by means of grafted shreds. In three others, matter scraped from the tumour was introduced into a deep wound of the neck. The results of these various experiments were entirely negative.

The account of this case has been obligingly communicated by M. Joffroy, whose name has been mentioned above.

\section{Aftedital Sorrities.}

\section{MEDICAL SOCIETY OF LONDON MONDAY, Nov. 2ND, 1868.}

Dr. P. W. Richardson, F.R.S., President, in the Charr.

MR. AlFRed Cooper showed a Calculus, which he had removed from the bladder of a boy aged twelve by the lateral operation of lithotomy. The point of interest was that the boy had been in the West London Hospital for seventeen days without showing a symptom of the presence of the foreign body.

Mr. Cooper also exhibited three Polypi of the Rectum, one of which he had removed from a child, one from a lady, and one from a gentleman. He had used the ligature in all the cases, and all had been mistaken for hæmorrhoids.

Mr. HEN RY SMITH showed a Stone weighing five and a half ounces, which be had removed from a woman's bladder. She had suffered eight or ten years from the disease. He extracted the calculus piecemeal by an incision made through the vagina and the neck of the bladder. The patient had since died.

Mr. SmrrH also exhibited a Stone from the female bladder, in which case he had performed the usual operation of lithotrity. At the second operation there was some difficulty in introducing the lithotrite, when the cause of the obstruction was discovered to be a large fragment, as big as a small walnut, which had become impacted in the urethra. This was removed by slightly incising the meatus, and the patient did well.

An animated discussion then followed on the question of dilating the urethra, Mr. Walter Coulson and Mr. Bryant being of opinion that rapid dilatation was seldom, if ever, followed. by incontinence, whereas slow or gradual dilatation was attended by that distressing sequel.

Mr. Henry LeE then read a paper on

THE MEDIO-LATERAL OPERATION OF LITHOTOMY.

The author described the way in which he now performs lithotomy, and which he has named the Medio-lateral Operation. He gave the particulars of three cases in which this operation had been performed. The first of these had occurred upwards of twelve months ago. The medio-lateral operation, he said, was performed in the following manner :-The patient is placed in the ordinary position for lithotomy, and a grooved staff having been introduced, an incision is then made in the median line of the perineum from before backward. This incision should extend through the posterior half of the perineum, terminating two or three lines in front of the anus. From this point the incision is continued for a quarter of a circle round the front and left side of the rectum. The finger of the left hand may then be put into the wound, and the rectum pressed back, whilst an additional touch or two with the knife separates it still further from the parts in front. The forefinger of the left hand is now passed into the rectum, and the knife, with its back towards the bowel, is passed at the posterior part of the central incision, and in the median line, into the membranous portion of the urethra. With the finger as a guide this is done with great ease and certainty. A bistoury or knife, with a probe as its extremity, is then passed into the same opening, and made to slide along the staff into the bladder. The blade of the knife is then directed towards the patient's left side, and somewhat backward, and as it is withdrawn the heel of 